Fetal Diagnosis and Therapy

\title{
First-Trimester Screening for Gestational Diabetes Mellitus Based on Maternal Characteristics and History
}

\author{
Argyro Syngelaki $^{\mathrm{a}, \mathrm{b}} \quad$ Alice Pastides $^{\mathrm{a}}$ Reena Kotecha ${ }^{\mathrm{a}} \quad$ Alan Wright $^{\mathrm{a}}$ \\ Ranjit Akolekar $^{\mathrm{a}, \mathrm{b}}$ Kypros H. Nicolaides ${ }^{\mathrm{a}, \mathrm{b}}$ \\ a Harris Birthright Research Centre for Fetal Medicine, King's College Hospital, London, and ${ }^{b}$ Department of \\ Fetal Medicine, Medway Maritime Hospital, Gillingham, UK
}

\section{Key Words}

Gestational diabetes mellitus · First-trimester screening ·

Pyramid of pregnancy care

\begin{abstract}
Objectives: To develop and validate a prediction model for gestational diabetes mellitus (GDM) at 11-13 weeks' gestation based on maternal characteristics and history and to compare its performance with the method recommended by the National Institute of Health and Care Excellence (NICE) and five other published prediction models. Methods: A predictive logistic regression model for GDM was developed from 1,827 cases (2.4\%) who developed GDM and 73,334 unaffected controls. A 5-fold cross-validation study was performed to validate this model and to compare its performance with those of the NICE guidelines and the previously published models. Results: In the logistic regression model, maternal age, weight, height, racial origin, family history of diabetes, use of ovulation drugs, birth weight, and previous history of GDM were found to be significant predictors of GDM. In screening for GDM in the 5-fold cross-validation study, detection rates (DRs) were higher $(p<0.0001)$ for the proposed model ( $D R=83.2 \%$ ) than for the NICE guidelines (DR $=77.5 \%$ ) for a false positive rate of approximately $40 \%$ (determined by NICE). The area under the receiver operating
\end{abstract}

characteristic curve of the new model was higher $(p<0.0001)$ than that of the previous five models (0.823 vs. $0.688-786)$. Conclusions: Early effective screening for GDM can be achieved based on maternal characteristics and history.

(c) 2014 S. Karger AG, Basel

\section{Introduction}

Gestational diabetes mellitus (GDM) is associated with an increased risk of maternal and perinatal shortand long-term complications [1-6]. The condition is diagnosed by a positive oral glucose tolerance test (OGTT), which is either carried out in all pregnant women [7] or in a selected group of women identified by their demographic characteristics and obstetric history as being at high risk for GDM [8]. In the UK, OGTT is offered to women with any one of the following risk factors: body mass index $(\mathrm{BMI})>30$, development of GDM in a previous pregnancy, previous delivery of a macrosomic baby $(\geq 4.5 \mathrm{~kg})$, first-degree relative with diabetes mellitus, or racial origin with a high prevalence of diabetes such as South Asian, African-Caribbean and Middle Eastern [8].

We have previously suggested that in screening for GDM it would be preferable to combine the various maternal factors into a multivariate logistic model, rather 
than treating each one as an independent screening test, as recommended by the National Institute of Health and Care Excellence (NICE) [9]. The multivariate model, which was derived from the study of 11,464 singleton pregnancies, including 297 (2.6\%) that developed GDM, detected about $75 \%$ of cases of GDM at a false positive rate (FPR) of $40 \%$.

The objectives of this study of 75,161 singleton pregnancies were to improve our previous prediction model for GDM and to compare its performance with the method recommended by NICE and that of other previously published clinical risk prediction models [9-13].

\section{Methods}

\section{Study Population}

The study population was derived from a prospective screening study on the early prediction of pregnancy complications in women attending for their routine first hospital pregnancy visit at King's College Hospital, London and Medway Maritime Hospital, Gillingham. At this visit, which is held at a gestation of $11+0$ to $13+6$ weeks, we record maternal characteristics and medical history and perform an ultrasound scan for the following purposes: (1) to confirm gestational age from the measurement of the fetal crown-rump length [14], (2) to diagnose any major fetal abnormalities [15] and (3) to screen for chromosomal abnormalities based on fetal nuchal translucency thickness and maternal serum pregnancy-associated plasma protein- $A$ and free $\beta$-human chorionic gonadotrophin $[16,17]$. Written informed consent was obtained from the women agreeing to participate in the study, which was approved by the NHS Research Ethics Committee.

The inclusion criterion for this study on screening for GDM was singleton pregnancy delivering a phenotypically normal neonate at or after 30 weeks' gestation. We excluded pregnancies with pre-pregnancy diabetes mellitus type 1 or 2 , those ending in termination, miscarriage, or delivery before 30 weeks because they may not have had screening and diagnosis of GDM.

Details of maternal characteristics and the findings of the assessment at 11-13 weeks were recorded in our database. Data on pregnancy outcome were obtained from the maternity computerized records or the general medical practitioners of the women and were also recorded in our database.

\section{Maternal History and Characteristics}

Patients were asked to complete a questionnaire on maternal age, racial origin (Caucasian, African, South Asian, East Asian, or mixed), cigarette smoking during pregnancy (yes or no), method of conception (spontaneous or assisted conception requiring the use of ovulation drugs), medical history (including pre-pregnancy diabetes mellitus type 1 or 2), family history of diabetes mellitus (first-, second- or third-degree relative with diabetes mellitus type 1 or 2), and obstetric history. The questionnaire was then reviewed by a doctor together with the patient and for the purpose of this study the women were classified as parous or nulliparous with no previous pregnancies at or beyond 24 weeks - if parous we recorded whether any of the previous pregnancies were complicated by
GDM (yes or no). The maternal weight and height were measured and the BMI was calculated in $\mathrm{kg} / \mathrm{m}^{2}$.

\section{Screening and Diagnosis of GDM}

In both participating hospitals, the diagnosis of GDM is based on a 75-gram OGTT performed at 24-28 weeks' gestation [18]. However, the screening policies for GDM differ between the two hospitals. In one unit, all women have a measurement of random plasma glucose taken at 24-28 weeks and OGTT is carried out if the concentration is $>6.7 \mathrm{mmol} / \mathrm{l}$. An OGTT is also performed if there is persistent glucosuria or polyhydramnios, or if the fetus becomes macrosomic. In the second unit, screening is based on risk factors, as recommended by NICE [8].

\section{Statistical Analysis}

As an exploratory measure, comparisons between the GDM and non-GDM groups were made for all variables of interest. The $\chi^{2}$ test was used to identify differences between levels of categorical variables for the outcome. Where appropriate, $t$ tests were used to assess differences between the two levels of the outcome for continuous variables; where not appropriate, the Mann-Whitney test was used.

A logistic regression model was fitted with GDM status as the outcome and various factors from the maternal characteristics and history as the predictors. Backwards elimination was employed as a variable selection technique. In this model, maternal age in years, weight in kilograms, height in centimetres, and birth weight $\mathrm{z}$ score of the last pregnancy with delivery at or beyond 24 weeks were treated as continuous variables. Maternal age at screening was centred at 35 years, maternal weight was centred at $69 \mathrm{~kg}$ and maternal height was centred at $164 \mathrm{~cm}$. The z-score is the difference in standard deviations between the observed and expected birth weight for gestational age [19]. Racial origin, method of conception, smoking, family history of diabetes, and previous GDM were treated as categorical variables.

A 5-fold cross-validation study was conducted to compare the performance of the new model with the NICE guidelines [8]. Essentially, the data were divided into five equal subgroups. The model was then fitted 5 times to different combinations of four of the five subgroups and validated in the remaining fifth of the data. Within the cross-validation study we inspected the detection rates (DRs) for prespecified FPR cut-offs and performed McNemar's test to provide evidence of any real differences between these DRs. $\mathrm{p}$ values from the 5 -fold cross-validation study were combined using Fisher's method.

There are five other published clinical risk prediction models for GDM [9-13]. In two of these models the authors combined maternal characteristics and obstetric history through multivariate logistic analysis to estimate the probability of GDM $[9,12]$, whereas the other three were based on a scoring system to determine the risk for GDM (table 1) [10,11,13]. The performance of each of these models was evaluated in our population by calculating the area under the receiver operating characteristic curve and the DR at FPRs of 10, 20 and $40 \%$. For the studies which used a scoring system instead of a multivariate regression model $[10,11$, 13] the DRs were obtained by linear interpolation between the two cut-offs, including the specified FPR; they could not be achieved without randomizing between cut-offs, which in practical terms is not feasible.

The statistical software package $\mathrm{R}$ was used for data analyses. 
Table 1. Risk factors and scoring systems for the prediction of gestational diabetes reported in the literature

\begin{tabular}{|c|c|c|}
\hline Study & Risk factors & Risk calculation \\
\hline Naylor et al. [10], 1997 & $\begin{array}{l}\text { Age in years } \\
\text { BMI } \\
\text { Race }\end{array}$ & $\begin{array}{l}<30: 0,31-34: 1,>35: 2 \\
<22: 0,22.1-25: 2,>25.1: 3 \\
\text { White or Black: 0, East Asian: 5, South Asian: } 2\end{array}$ \\
\hline Caliskan et al. [11], 2004 & $\begin{array}{l}\text { Age in years } \\
\text { BMI } \\
\text { 1st-degree relative with DM } \\
\text { Previous } \mathrm{BW}>4,000 \mathrm{~g} \\
\text { History of adverse outcome }\end{array}$ & $\begin{array}{l}<25: 0,>25: 1 \\
<25: 0,>25: 1 \\
\text { No: } 0 \text {, yes: } 1 \\
\text { No: } 0 \text {, yes: } 1 \\
\text { No: } 0 \text {, yes: } 1\end{array}$ \\
\hline van Leeuwen et al. [12], 2010 & $\begin{array}{l}\text { BMI, race, } 1 \text { st- or } 2 \text { nd-degree } \\
\text { relative with DM, previous } \\
\text { GDM }\end{array}$ & $\begin{array}{l}\text { Probability of GDM }=1 /[1+\exp (-\mathrm{b})] \text {, in which } \mathrm{b} \text { is calculated as } \\
{[-6.1+(0.83 \cdot \text { non-Caucasian ethnicity })+(0.57 \cdot \text { positive family }} \\
\text { history of diabetes mellitus })-(0.67 \cdot \text { multipara without history of } \\
\text { GDM })+(0.5 \cdot \text { multipara with history of GDM })+(0.13 \cdot \text { BMI })]\end{array}$ \\
\hline Nanda et al. [9], 2011 & $\begin{array}{l}\text { Age in years, BMI, race, } \\
\text { previous GDM, previous } \\
\text { BW }>90 \text { th centile }\end{array}$ & $\begin{array}{l}\text { Probability of GDM }=1 /[1+\exp (-\mathrm{b})] \text {, in which b is calculated as } \\
{[-8.68947+(0.05365 \cdot \text { age })+(0.10852 \cdot \mathrm{BMI})+(1.00312 \cdot \text { South }} \\
\text { Asian })+(0.88785 \cdot \text { East Asian })+(3.72259 \cdot \text { previous GDM })+ \\
(0.67673 \cdot \text { previous BW }>90 \text { th centile })]\end{array}$ \\
\hline
\end{tabular}

History of adverse outcome includes recurrent spontaneous abortions, previous fetal anomaly or previous fetal death $>20$ weeks of gestation. DM = Diabetes mellitus; BW = birth weight.

\section{Results}

\section{Screening Population}

During the period between March 2006 and July 2013 there were 75,161 singleton pregnancies which fulfilled the inclusion criteria. These included 1,827 (2.4\%) that developed GDM and 73,334 that were unaffected by diabetes. The maternal and pregnancy characteristics of the GDM and non-GDM groups are compared in table 2. In the GDM group, the women tended to be older, heavier and shorter, and there was a higher proportion of African, South Asian and East Asian racial origin, conceptions with ovulation drugs, history of first- or second-degree relative with diabetes, and previous pregnancies complicated by GDM or deliveries of macrosomic neonates.

\section{Screening for GDM by the NICE Guidelines}

In the GDM group, compared with those without GDM, there was a higher incidence of BMI $>30$ (44.4 vs. $16.6 \%$ ), previous GDM (26.2 vs. $0.6 \%$ ), previous delivery of neonate weighing $\geq 4.5 \mathrm{~kg}$ ( $3.0 \mathrm{vs.} 0.7 \%$ ), first-degree relative with diabetes mellitus (29.3 vs. $13.0 \%$ ), and women of South Asian or African-Caribbean racial origin (33.6 vs. $20.4 \%$; table 1 ). At least one of these NICE criteria was fulfilled by 1,425 of the 1,827 cases with GDM (78.0\%) and by 29,320 of the 73,334 cases without GDM (40.0\%).

\section{New Logistic Regression Model}

The logistic regression model, as fitted to the 1,827 cases with GDM and 73,334 controls, is summarized in table 3. In this model, the women with and without previous history of GDM were treated differently because in the former there is a high risk of recurrence, and the contribution of other risk factors other than weight is negligible. In nulliparous women and in parous women with no previous history of GDM, significant contributions for the prediction of GDM were provided from maternal age, weight, height, racial origin, first- and second-degree family history of diabetes mellitus, conception by use of ovulation induction drugs, and previous birth weight $\mathrm{z}$ score. The relationships between maternal weight, height,
16

Fetal Diagn Ther 2015;38:14-21 DOI: $10.1159 / 000369970$
Syngelaki/Pastides/Kotecha/Wright/ Akolekar/Nicolaides 
Table 2. Maternal and pregnancy characteristics in the screening population

\begin{tabular}{|c|c|c|c|}
\hline Variables & GDM $(\mathrm{n}=1,827)$ & Unaffected $(\mathrm{n}=73,334)$ & $\mathrm{p}$ value \\
\hline Maternal age, years & $33.1(29.2-36.9)$ & $30.7(36.1-34.6)$ & $<0.001$ \\
\hline Maternal weight, kg & $76.7(65.0-91.4)$ & $66.0(59.0-76.0)$ & $<0.001$ \\
\hline Maternal height, $\mathrm{cm}$ & $162.6(158.0-167.7)$ & $164.6(160.0-169.0)$ & $<0.001$ \\
\hline BMI & $29.0(24.6-33.9)$ & $24.3(21.9-28)$ & $<0.001$ \\
\hline Racial origin & & & $<0.001$ \\
\hline South Asian & $168(9.2)$ & $3,105(4.2)$ & \\
\hline East Asian & $82(4.5)$ & $1,539(2.1)$ & \\
\hline Mixed & $38(2.1)$ & $1,778(2.4)$ & \\
\hline Cigarette smokers & $139(7.6)$ & $7,499(10.2)$ & $<0.001$ \\
\hline Conception & & & $<0.001$ \\
\hline Spontaneous & $1,732(94.8)$ & $70,909(96.7)$ & \\
\hline 2 nd-degree relative & $189(10.3)$ & $6,153(8.4)$ & \\
\hline 3 rd-degree relative & $51(2.8)$ & $1,860(2.5)$ & \\
\hline Parity & & & $<0.001$ \\
\hline Nulliparous & $822(45.0)$ & $39,761(54.2)$ & \\
\hline Parous with previous GDM & $479(26.2)$ & $427(0.6)$ & \\
\hline Parous with no previous GDM & $526(28.8)$ & $33,146(45.2)$ & \\
\hline Parous previous birth weight $\mathrm{z}$-score & $0.393 \pm 1.335$ & $-0.0359 \pm 1.114$ & $<0.001$ \\
\hline Gestation at delivery, weeks & $38.7(38.1-39.4)$ & $40.1(39.1-41.0)$ & $<0.001$ \\
\hline Birth weight, $g$ & $3,330(3,005-3,685)$ & $3,400(3,080-3,730)$ & $<0.001$ \\
\hline
\end{tabular}

Values are presented as medians (IQR), numbers (percentages) or means \pm SD, as appropriate. Comparison between outcome groups by Mann-Whitney $U$ test for continuous variables and $\chi^{2}$ test for categorical variables. Significance value $p<0.05$. Wilcoxon rank-sum test performed on gestation at delivery in weeks due to skewed distribution.

age, and previous birth weight $\mathrm{z}$-score and the probability of GDM are shown in figures 1 and 2.

\section{Comparison of Performance of the New Model with \\ NICE Guidelines}

Comparisons of DRs for the new model and the NICE guidelines [8] from the 5-fold cross-validation study are shown in table 4 . In each of the five groups, the DR for the given FPR was higher with the new model than with the NICE guidelines. The $\mathrm{p}$ values from McNemar's test were $0.0007,0.2718,0.1344,0.0003$, and 0.1416 , respectively, and when these values were combined using Fisher's method the $\mathrm{p}$ value was $<0.0001$.

\section{Comparison of Performance of the New Model with}

Other Risk Prediction Models

The performance of screening for GDM by five published clinical risk prediction models [9-13] was com- pared with that of our new model, as shown in figure 3 and table 5. The area under the receiver operating characteristic curve in our model was higher than that of each of the previous models - in three of the models, the performance of screening for GDM in our study population was similar to the reported performance in the relevant publication $[9,10,12]$, in one it was better $[13]$ and in another model it was worse [11].

\section{Discussion}

\section{Main Findings of the Study}

Screening for GDM by maternal characteristics and obstetric history is associated with a higher DR for a given FPR if the maternal factors are combined into a multivariate logistic model, rather than treating each one as an independent screening test, as recommended by NICE 
Table 3. Logistic regression analysis to determine factors defining the a priori risk for the prediction of GDM from maternal history and characteristics

\begin{tabular}{|c|c|c|c|c|c|c|}
\hline Term & Odds ratio & LCI & UCI & Estimate & SE & $\mathrm{p}$ value \\
\hline Intercept & - & - & - & -4.0050 & 0.0489 & $<0.0001$ \\
\hline Previous GDM & 50.4447 & 42.1338 & 60.3948 & 3.9209 & 0.0919 & $<0.0001$ \\
\hline \multicolumn{7}{|l|}{ Nulliparous or parous with no previous GDM } \\
\hline Parous: no previous GDM & 0.4545 & 0.4026 & 0.5131 & -0.7885 & 0.0618 & $<0.0001$ \\
\hline Age (35 years) & 1.0841 & 1.0734 & 1.0948 & 0.0807 & 0.0050 & $<0.0001$ \\
\hline Height $(164 \mathrm{~cm})$ & 0.9426 & 0.9341 & 0.9512 & -0.0591 & 0.0046 & $<0.0001$ \\
\hline 1st-degree relative with DM & 2.5427 & 2.2387 & 2.8879 & 0.9332 & 0.0650 & $<0.0001$ \\
\hline 2nd-degree relative with DM & 1.7984 & 1.4940 & 2.1650 & 0.5869 & 0.0946 & $<0.0001$ \\
\hline Ovulation drugs & 1.6019 & 1.1118 & 2.3080 & 0.4712 & 0.1863 & 0.0114 \\
\hline Afro-Caribbean racial origin & 1.5780 & 1.3756 & 1.8102 & 0.4562 & 0.0700 & $<0.0001$ \\
\hline East Asian racial origin & 2.9232 & 2.2381 & 3.8181 & 1.0727 & 0.1363 & $<0.0001$ \\
\hline
\end{tabular}

Data in parentheses indicate the levels at which age, weight and height were centred. LCI = Lower confidence interval; UCI = upper confidence interval; $\mathrm{SE}=$ standard error; $\mathrm{DM}=$ diabetes mellitus.

[9]. Screening by the new model can predict 55, 68 and $84 \%$ of cases of GDM at FPRs of 10, 20 and 40\%, respectively. The DR by the NICE guidelines is $78 \%$ at an FPR of $40 \%$.

In the new model, the predictors of GDM are previous history of GDM, family history of first- or second-degree relative with diabetes mellitus, maternal age, weight, height, racial origin, method of conception, and birth weight of the neonate in the last pregnancy. We found that for women with a previous history of GDM the probability of developing GDM in the current pregnancy was very high and the contribution of risk factors other than weight was negligible.

\section{Strengths and Limitations}

The major strengths of the study are, firstly, the prospective examination of a large number of pregnancies and, secondly, the use of a multivariate logistic model to identify the significant factors and to define their individual contribution in the prediction of GDM. We examined about 75,000 women with singleton pregnancies within a narrow gestational age range at 11-13 weeks, which was accurately determined by the sonographic measurement of the fetal crown-rump length. We asked specific questions to identify known factors associated with GDM and measured their weight and height. The use of a multivariate logistic model, in which all possible

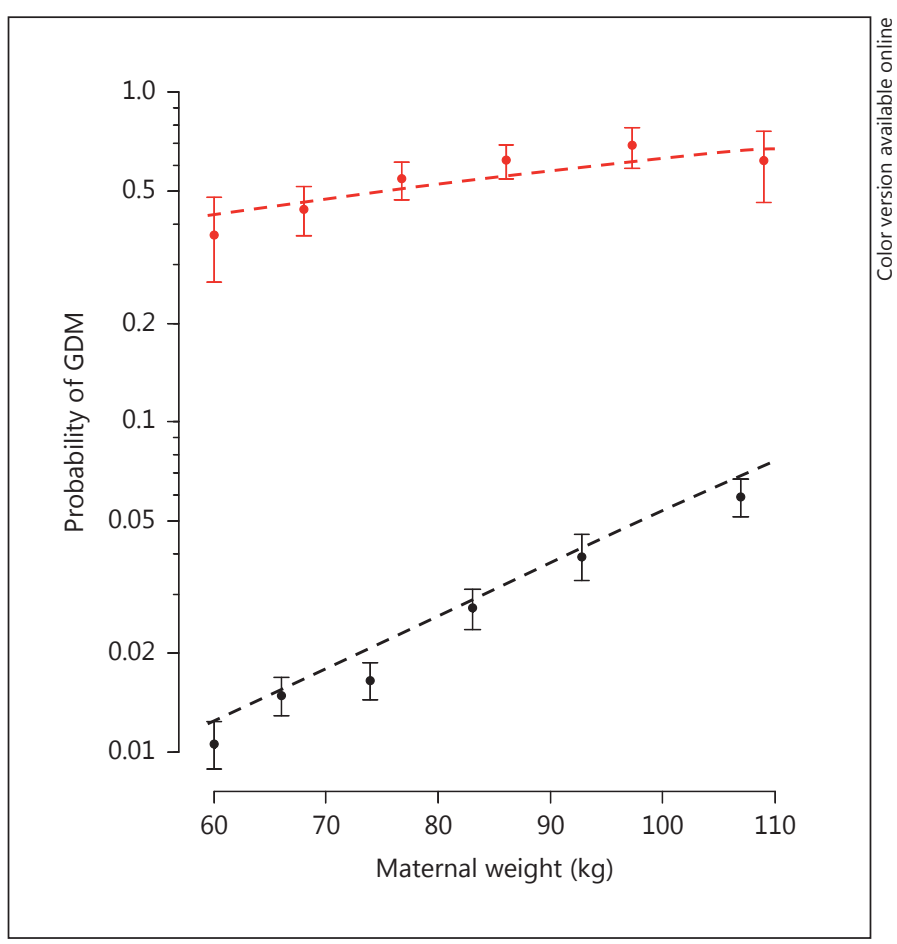

Fig. 1. Relationship between maternal weight and probability of developing GDM in women with (red lines; colour refers to the online version only) and without (black lines) a history of previous GDM. The interrupted lines represent the fitted probabilities for GDM and the circles (with 95\% confidence intervals) represent the observed proportions of GDM for maternal weight groups.
18

Fetal Diagn Ther 2015;38:14-21 DOI: $10.1159 / 000369970$
Syngelaki/Pastides/Kotecha/Wright/ Akolekar/Nicolaides 


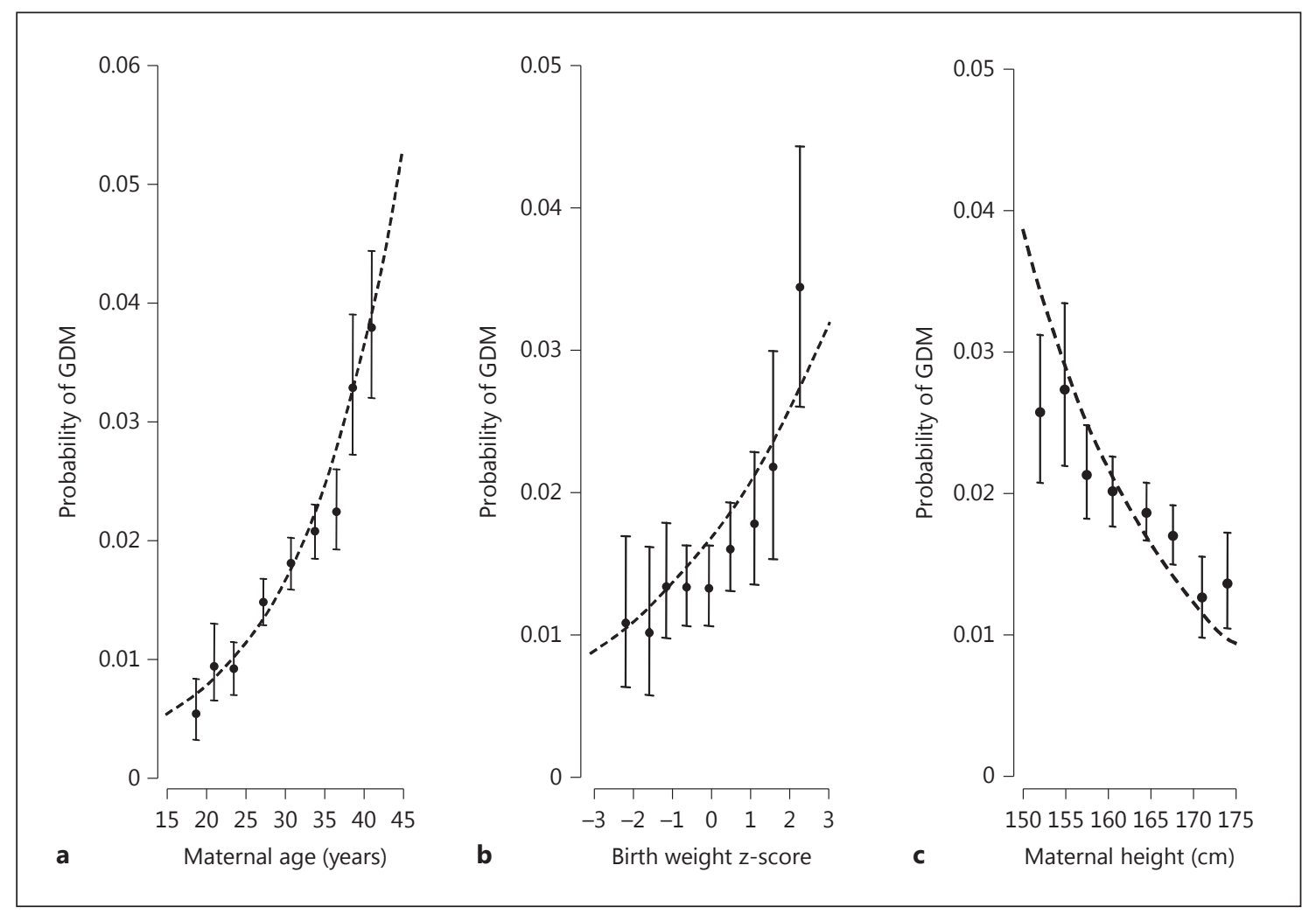

Fig. 2. Relationship between maternal age (a), previous birth weight z-score (b) and maternal height (c) and the probability of developing GDM in women without a history of previous GDM. The interrupted lines represent the fitted probabilities for GDM and the circles (with 95\% confidence intervals) represent the observed proportions of GDM for maternal age, height and birth weight z-score groups.

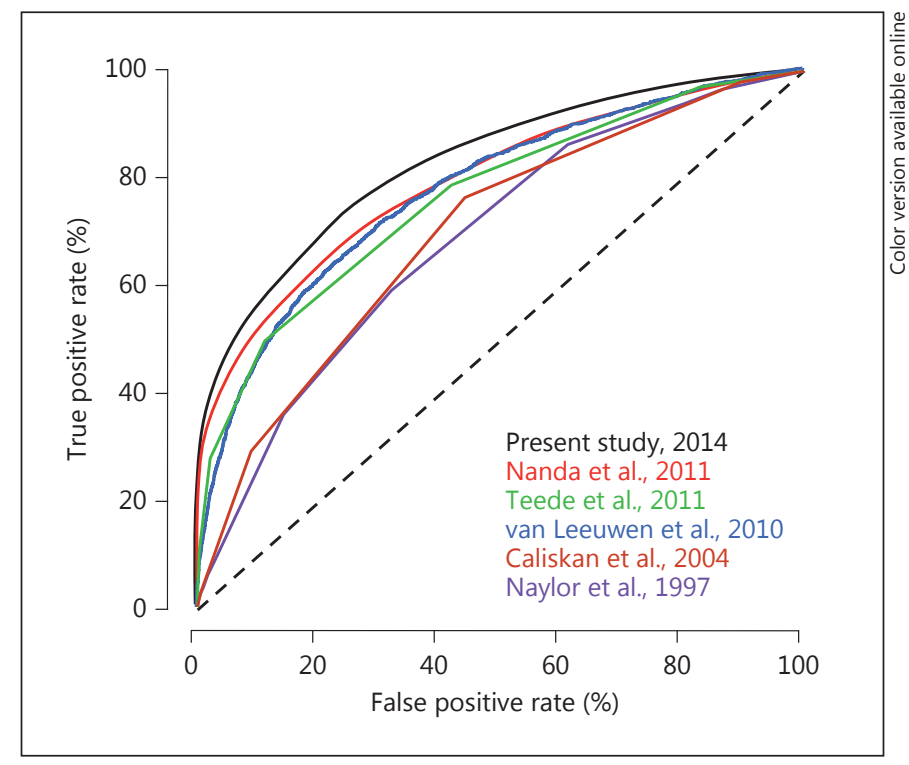

Fig. 3. Relationship between true and false positive rates in screening for GDM in the new model and in five previously published clinical risk prediction models.

Early Screening for Gestational Diabetes Mellitus
Table 4. Comparisons of detection rates for the new model of screening for GDM and the NICE guidelines from the 5-fold crossvalidation study

\begin{tabular}{lllll}
\hline Group & FPR, \% & \multicolumn{2}{c}{ Detection rate, $\%$} & p value \\
\cline { 3 - 4 } & & \multicolumn{3}{c}{$\begin{array}{l}\text { NICE model } \\
\text { guidelines }\end{array}$} \\
\hline 1 & 40.01 & 86.36 & 78.13 & 0.0007 \\
2 & 39.53 & 82.03 & 78.84 & 0.2718 \\
3 & 39.37 & 79.73 & 76.00 & 0.1344 \\
4 & 38.83 & 84.85 & 76.86 & 0.0003 \\
5 & 39.13 & 82.91 & 77.78 & 0.1416 \\
\hline
\end{tabular}

The FPR in each group was that derived from the NICE guidelines. When the $\mathrm{p}$ values (McNemar's test) were combined using Fisher's method the $\mathrm{p}$ value was $<0.0001$.
Fetal Diagn Ther 2015;38:14-21 DOI: $10.1159 / 000369970$ 
Table 5. Comparison of performance of screening for GDM of the new model with that of previously published models or risk scoring systems

\begin{tabular}{|c|c|c|c|c|c|}
\hline Prediction model & Derived AUROC & Original AUROC & \multicolumn{3}{|c|}{ Detection rate at FPR of: } \\
\hline Naylor et al. [10], 1997 & $0.688(0.684-0.691)^{1}$ & $0.733(0.711-0.755)$ & 27 & 44 & 67 \\
\hline Caliskan et al. [11], 2004 & $0.699(0.696-0.703)^{1}$ & $0.832(0.793-0.867)^{3}$ & 31 & 44 & 71 \\
\hline van Leeuwen et al. [12], 2010 & $0.772(0.769-0.775)^{1}$ & $0.770(0.690-0.850)$ & 46 & 61 & 79 \\
\hline
\end{tabular}

Values in parentheses are 95\% CI. Detection rates are given as percentages. The derived area under the receiver operating characteristic (AUROC) curve is the value obtained using the model or risk scoring system in the current study population. The original AUROC curve is the value reported or calculated by ourselves in the original study population.

${ }^{1}$ The AUROC curve in these models was significantly lower $(\mathrm{p}<0.0001)$ than in the new model.

${ }^{2}$ The AUROC curve in the original publication was significantly lower $(\mathrm{p}=0.043)$ than the value obtained from applying the model in the current study population.

${ }^{3}$ The AUROC curve in the original publication was significantly higher $(\mathrm{p}=0.008)$ than the value obtained from applying the model in the current study population.

predictors are treated as continuous variables, can define the individual patient risk for GDM, allowing for different management protocols and future research based on selected probability cut-offs.

A limitation of the study relates to the method of identifying the GDM-affected pregnancies. The diagnostic OGTT was not carried out in all pregnancies, as recommended by the International Association of Diabetes and Pregnancy Study Groups [7], but only in those with risk factors as recommended by NICE [8] or abnormal results of a random blood glucose level at 24-28 weeks' gestation. It is, therefore, possible that some of the women included in our non-GDM group actually had GDM and that the performance of screening of our method was overestimated. However, the same would be true for the method recommended by NICE.

Another potential limitation of the study is that the performance of screening by a model derived from the same study population was overestimated due to overfitting of the data. We have addressed this issue by the 5-fold cross-validation study.

\section{Comparison with Findings from Previous Studies}

In our study the risk for GDM increased with maternal age and weight, and was higher in women of Afro-Caribbean and Asian racial origin than in Caucasians, in those with a family history of diabetes, in those who conceived with the use of ovulation induction drugs, and in those with a previous pregnancy complicated by GDM or macrosomia. Most of these risk factors have also been highlighted in previous clinical risk prediction models for GDM [8-13].

Screening by the NICE guidelines [8] and most previous risk prediction models [10-13] assumes a step function for all continuous measurements. However, we demonstrated that the observed proportions of GDM by maternal age, weight, height, and birth weight do not follow a step function but a smooth curve. Consequently, in our model these factors are treated as continuous rather than categorical variables.

The study populations for the development of the five previous prediction models for GDM varied between 995 and 4,612 patients and differed in maternal characteristics, diagnostic criteria for GDM and prevalence of GDM (2.4-8.3\%) [9-13]. Nevertheless, the external validation undertaken in our large population demonstrated that the performance of most models was similar to that in the original studies $[9,10,12]$. The performance of one of the models was better [13] and that of another was worse [11] in our population than in the original studies. Similar results were obtained in a previous study of 7,929 pregnant women in which four [10-13] of the five prediction models for GDM were evaluated [20]. 


\section{Conclusion}

The screening and diagnosis of GDM is traditionally delayed until the late second or early third trimester of pregnancy, because the diabetogenic effects of pregnancy increase with gestation and, therefore, delayed testing maximizes the DR. An alternative approach is to undertake earlier testing and adjust the traditional criteria of the tests with the rationale that early identification of the high-risk group is likely to improve pregnancy outcome because with appropriate dietary advice and pharmacological interventions the incidence of the disease and associated maternal and perinatal complications could po- tentially be reduced [21]. In this respect, our model provides an effective method of early screening for GDM. Additionally, the model allows the estimation of the patient-specific a priori risk of GDM, which could be combined with potentially useful biomarkers such as maternal serum adiponectin, visfatin, tissue plasminogen activator, and sex hormone-binding globulin $[9,22,23]$, with further improvement in the performance of screening.

\section{Acknowledgement}

This study was supported by grants from the Fetal Medicine Foundation (charity No: 1037116).

\section{References}

1 Casey BM, Lucas MJ, Mcintire DD, Leveno $\mathrm{KJ}$ : Pregnancy outcomes in women with gestational diabetes compared with the general obstetric population. Obstet Gynecol 1997; 90:869-873.

-2 Crowther CA, Hiller JE, Moss JR, McPhee AJ, Jeffries WS, Robinson JS: Effect of treatment of gestational diabetes on pregnancy outcomes. Australian Carbohydrate Intolerance Study in Pregnant Women (ACHOIS) Trial Group. N Engl J Med 2005;352:2477-2486.

-3 Clausen TD, Mathiesen ER, Hansen T, Pedersen O, Jensen DM, Lauenborg J, Damm P: High prevalence of type 2 diabetes and prediabetes in adult offspring of women with gestational diabetes mellitus or type 1 diabetes: the role of intrauterine hyperglycemia. Diabetes Care 2008;31:340-346.

$\checkmark 4$ HAPO Study Cooperative Research Group; Metzger BE, Lowe LP, Dyer AR, Trimble ER, Chaovarindr U, Coustan DR, Hadden DR, McCance DR, Hod M,McIntyre HD, Oats JJ, Persson B, Rogers MS, Sacks DA: Hyperglycemia and adverse pregnancy outcomes. N Engl J Med 2008;358:1991-2002.

$\checkmark 5$ Feig DS, Zinman B, Wang X, Hux JE: Risk of development of diabetes mellitus after diagnosis of gestational diabetes. CMAJ 2008;179: 229-234.

$>6$ Bellamy L, Casas JP, Hingorani AD, Williams D: Type 2 diabetes mellitus after gestational diabetes: a systematic review and meta-analysis. Lancet 2009;373:1773-1779.

7 International Association of Diabetes and Pregnancy Study Groups Consensus Panel; Metzger BE, Gabbe SG, Persson B, Buchanan TA, Catalano PA, Damm P,Dyer AR, Leiva Ad, Hod M, Kitzmiler JL, Lowe LP, McIntyre HD, Oats JJ, Omori Y, Schmidt MI: International Association of Diabetes and Pregnancy Study Groups recommendations on the diagnosis and classification of hyperglycemia in pregnancy. Diabetes Care 2010;33:676-682.
-8 National Collaborating Centre for Women's and Children's Health: Diabetes in pregnancy: management of diabetes and its complications from pre-conception to the postnatal period. Clinical Guideline 63. London, National Institute for Health and Clinical Excellence, 2008. www.nice.org.uk/CG063fullguideline.

-9 Nanda S, Savvidou M, Syngelaki A, Akolekar $\mathrm{R}$, Nicolaides KH: Prediction of gestational diabetes mellitus by maternal factors and biomarkers at 11 to 13 weeks. Prenat Diagn 2011; 31:135-141.

10 Naylor CD, Sermer M, Chen E, Farine D: Selective screening for gestational diabetes mellitus. Toronto Trihospital Gestational Diabetes Project Investigators. N Engl J Med 1997; 337:1591-1596

11 Caliskan E, Kayikcioglu F, Ozturk N, Koc S, Haberal A: A population-based risk factor scoring will decrease unnecessary testing for the diagnosis of gestational diabetes mellitus. Acta Obstet Gynecol Scand 2004;83:524-530.

12 van Leeuwen M, Opmeer BC, Zweers EJ, van Ballegooie E, ter Brugge HG, de Valk HW, Visser GH, Mol BW: Estimating the risk of gestational diabetes mellitus: a clinical prediction model based on patient characteristics and medical history. BJOG 2010;117:69-75.

$\checkmark 13$ Teede HJ, Harrison CL, Teh WT, Paul E, Allan CA: Gestational diabetes: development of an early risk prediction tool to facilitate opportunities for prevention. Aust NZ J Obstet Gynaecol 2011;51:499-504.

14 Robinson HP, Fleming JE: A critical evaluation of sonar crown rump length measurements. Br J Obstet Gynaecol 1975;82:702-710.

15 Syngelaki A, Chelemen T, Dagklis T, Allan L, Nicolaides KH: Challenges in the diagnosis of fetal non-chromosomal abnormalities at 1113 weeks. Prenat Diagn 2011;31:90-102.
16 Nicolaides KH: Screening for fetal aneuploidies at 11 to 13 weeks. Prenat Diagn 2011;31: $7-15$.

17 Wright D, Syngelaki A, Bradbury I, Akolekar R, Nicolaides KH: First-trimester screening for trisomies 21, 18 and 13 by ultrasound and biochemical testing. Fetal Diagn Ther 2014; 35:118-126.

18 World Health Organization Department of Noncommunicable Disease Surveillance: Definition, diagnosis and classification of diabetes mellitus and its complications. Report of a WHO consultation. Part 1. Diagnosis and classification of diabetes mellitus. Geneva World Health Organization, 1999.

$\checkmark 19$ Poon LC, Volpe N, Muto B, Syngelaki A, Nicolaides KH: Birthweight with gestation and maternal characteristics in live births and stillbirths. Fetal Diagn Ther 2012;32:156-165.

20 Theriault S, Forest JC, Masse J, Giguere Y: Validation of early risk-prediction models for gestational diabetes based on clinical characteristics. Diabetes Res Clin Pract 2014;103: 419-425.

-21 Plasencia W, Garcia R, Pereira S, Akolekar R, Nicolaides KH: Criteria for screening and diagnosis of gestational diabetes mellitus in the first trimester of pregnancy. Fetal Diagn Ther 2011;30:108-115.

22 Ferreira AF, Rezende JC, Vaikousi E, Akolekar R, Nicolaides KH: Maternal serum visfatin at 11-13 weeks of gestation in gestational diabetes mellitus. Clin Chem 2011;57:609613.

23 Savvidou M, Nelson SM, Makgoba M, Messow CM, Sattar N, Nicolaides K: First-trimester prediction of gestational diabetes mellitus: examining the potential of combining maternal characteristics and laboratory measures. Diabetes 2010;59:3017-3022. 